\title{
An Unusual Case of Resistant Hepatic Hydrothorax Treated with Octreotide Infusion
}

\author{
Mehrdad Ghahremani-Ghajar, Mostafa Tabassomi, Scott Harada, Jose \\ Joseph Vempilly
}

\begin{abstract}
Hepatic hydrothorax is an uncommon clinical problem observed in patients with end stage liver disease and portal hypertension. The pathogenesis of pleural effusion in this condition is thought to involve the movement of ascitic fluid across diaphragmatic defects into pleural cavity facilitated by a negative pleural pressure. Therefore, tube thoracostomy is not considered to be a definitive treatment option in the management of hepatic hydrothorax. We present a case of massive pleural effusion secondary to hepatic hydrothorax not responding to conventional treatment successfully treated with intravenous infusion of octreotide.
\end{abstract}

Key words: TIPS: Transjugular Intrahepatic Portosystemic Shunt, tPA: Tissue Plasminogen Activator

\section{INTRODUCTION}

Hepatic hydrothorax is characterized as pleural effusion occurring in patients with end stage liver disease and portal hypertension in the absence of primary cardiac or pulmonary disease. It is an uncommon manifestation and is estimated to occur in $5-12 \%$ of cirrhotics. 1 Hepatic hydrothorax usually accumulates in the right pleural space and can occur without concurrent ascites. 1 The mechanism for pleural fluid formation in hepatic hydrothorax is proposed to involve the passive movement of ascitic fluid from peritoneal cavity to pleural space across the diaphragmatic defects facilitated by a negative intrathoracic pressure.2 Treatment options for hepatic hydrothorax that is refractory to diuresis and salt restriction are limited to serial thoracentesis, pleurodesis, thoracoscopic repair of diaphragmatic defect and transjugular intrahepatic portosystemic shunt (TIPS) placement. However, limited numbers of case reports have documented

\footnotetext{
To whom correspondence should be addressed:

Jose Joseph Vempilly, MD, FCCP

UCSF Fresno Center for Medical Education and Research

155 N. Fresno Street

Fresno, CA 93701-2302

jjoseph@fresno.ucsf.edu
}

resolution of hepatic hydrothorax with octreotide (Sandostatin; Novartis Pharma Stein AG; Stein, Switzerland) infusion. 3 We report a unique case of an abrupt onset hepatic hydrothorax in a patient with tuberculous pleurisy following a thoracoscopic fibrinolysis of pleural adhesions and its complete resolution with octreotide infusion.

\section{CASE}

A 47-year-old man with alcoholic liver disease was admitted for syncopal attack, due to severe postural drop in blood pressure from gastrointestinal blood loss. Three days following admission, patient was transferred to medical ICU for respiratory failure secondary to delirium from alcohol withdrawal and a massive left pleural effusion [Fig 1]. A chest tube was placed to drain the left effusion in ICU. Pleural fluid analysis revealed a lymphocyte predominant exudate. Pleural fluid Gram stain and microbiology studies were unremarkable. A Quantiferon-TB test was found to be positive. Four-drug anti-tuberculous regimen was initiated due to high clinical suspicion for tuberculous pleurisy. However, a definite diagnosis of tuberculous pleurisy was necessary to continue 


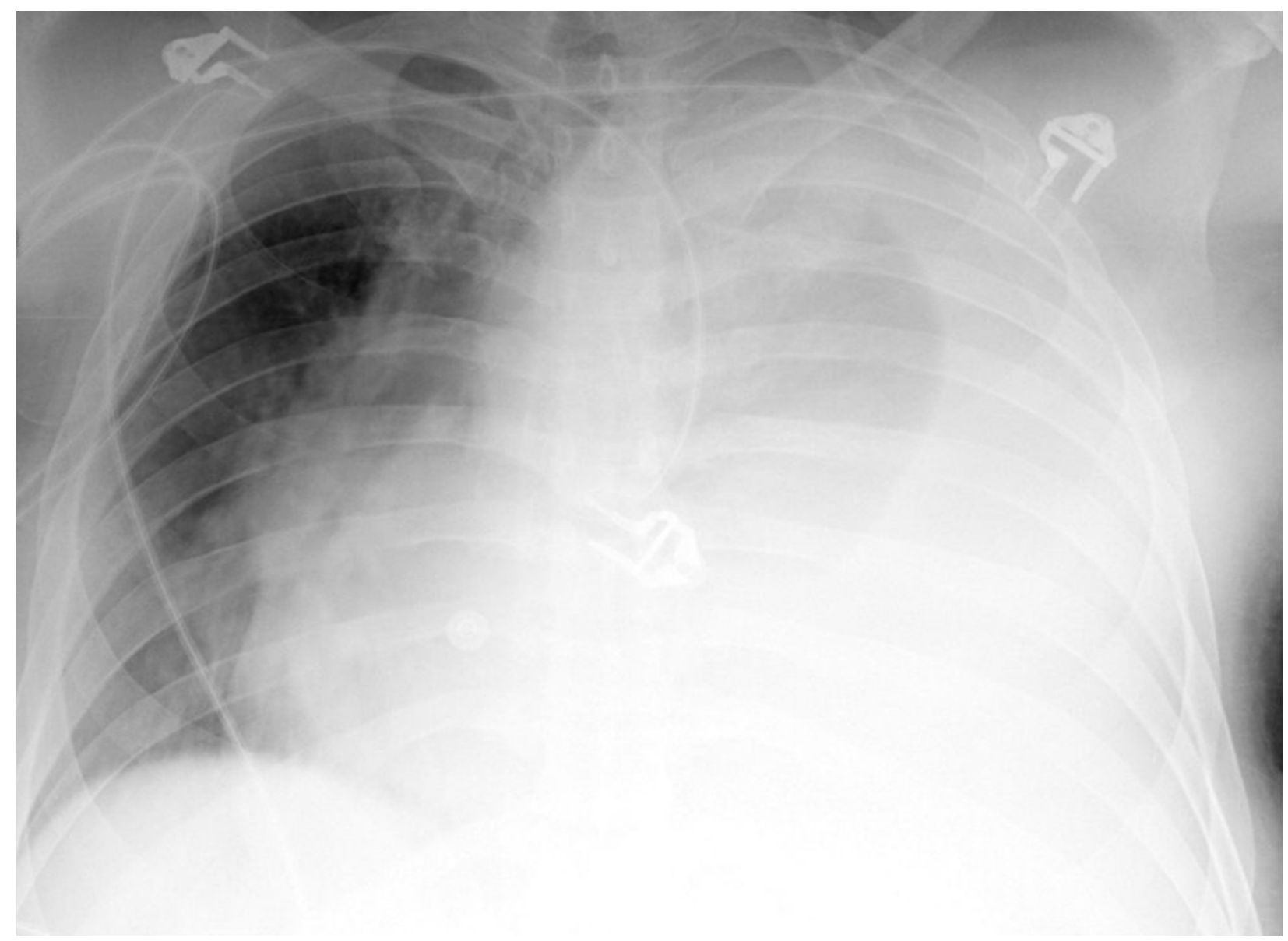

Figure 1: Chest x-ray on admission showing massive left pleural effusion.

the four drugs therapy in a patient with chronic liver disease. Therefore, a diagnostic pleuroscopy was done on left side which confirmed the diagnosis.

After obtaining a pleural biopsy, $100 \mathrm{ml}$ of tissue plasminogen activator (tPA) was instilled into the pleural cavity to breakdown basal pleural adhesions. Post-extubation, the 24 hour pleural fluid drainage was minimal with good expansion of the lung. However, a day after thoracoscopy, there was an abrupt increase in pleural fluid output; totaling $2150 \mathrm{ml} /$ day [Fig 2]. A repeat pleural fluid analysis showed a lymphocyte predominant transudative pleural effusion. A clinical diagnosis of hepatic hydrothorax was made based on history of cirrhosis and absence of heart failure or nephrotic syndrome. Therefore, diuresis with a loop diuretic was initiated with no reduction in daily pleural fluid drainage. Due to minimal response, a trial of octreotide infusion was initiated at $50 \mathrm{ug} / \mathrm{kg} / \mathrm{hour}$. Thereafter, pleural fluid drainage reduced from $2000 \mathrm{ml} /$ day to $600 \mathrm{ml}$ within a day. As the initial response was satisfactory, the octreotide infusion was continued for another 48 hours with complete resolution of plural fluid drainage by third day. Subsequently the chest tube was removed and patient discharged with anti-tuberculous therapy. A follow-up chest $x$-ray at three months showed no recurrence of pleural effusion [Fig 3].

\section{DISCUSSION}

This was a case of lymphocyte rich exudative pleural effusion due to tuberculous pleurisy that resolved with chest tube drainage and antituberculous therapy. Nevertheless, an increase in the size of pleural effusion while on anti-tuberculous therapy was considered a possible immune exacerbation of tuberculous pleurisy observed in some patients.4 However, an abrupt onset increase in fluid drainage as well as a change of pleural fluid chemistry from an exudate into a lymphocyte predominant transudate, made this possibility less likely. Furthermore, there was no evidence to support a diagnosis of heart failure associated pleural effusion. An iatrogenic diaphragmatic defect post thoracoscopic tPA seemed likely. Lack of response to conventional therapy prompted 


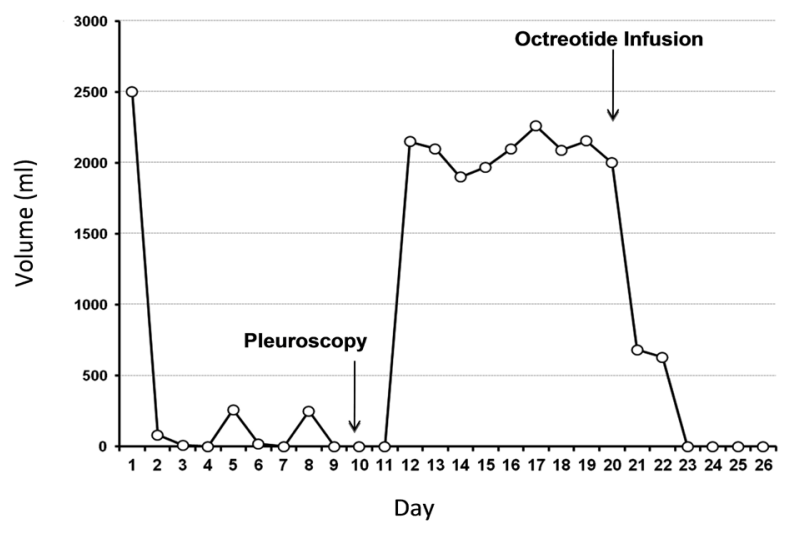

Figure 2: Pleural fluid drainage was negligible following initial chest tube placement. Post tPA administration for adhesion lysis, there was an abrupt increase in chest tube drainage, which did not respond to diuretic therapy. Following octreotide administration there was an abrupt drop in chest tube drainage.

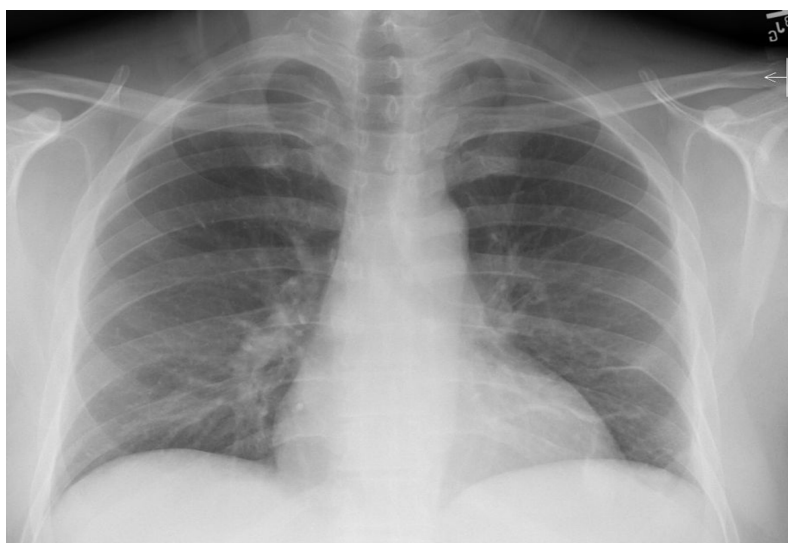

Figure 3: Chest x-ray follow-up in three months.

a trial of octreoide infusion, based on a limited number of previous case reports indicating efficacy of octreotide. An abrupt decrease in pleural fluid drainage with complete resolution within 72 hours following octreotide infusion indicated a therapeutic response. In this context, TIPS procedure has been shown to decrease the portal vein pressure and thereby reduce ascites and pleural effusion. 5 Therefore; it is possible that octreotide therapy by reducing the portal pressure may have provided the same benefit as TIPS leading to the resolution of ascites and hepatic hydrothorax in this patient. As the clinical evidence for octreotide in the treatment of hepatic hydrothorax mounts, a multicenter controlled trial of octreotide is warranted.

\section{REFERENCES}

1. Lazaridis KN, Frank JW, Krowka MJ, Kamath PS. Hepatic hydrothorax: pathogenesis, diagnosis, and management. Am. J. Med. 1999; 107: 262-7.

2. Nakamura A, Kojima $\mathrm{Y}$, Ohmi $\mathrm{H}$, et al. Peritonealpleural communications in hepatic hydrothorax demonstrated by thoracoscopy. Chest. 1996; 109:579-581.

3. Pfammatter R, Quattropani C, Reichen J, Göke $\mathrm{B}$,Wagner AC. Treatment of hepatic hydrothorax and reduction of chest tube output with octreotide. European Journal Of Gastroenterology \& Hepatology. 2001; 13:977-980.

4. Gupta R, DixitR, Purohit S, SaxenaA. Development of Pleural Effusion in Patients During Anti-Tuberculous Chemotherapy: Analysis of Twenty-Nine Cases with Review of Literature. Indian J Chest Dis Allied Sci. 2000; 42: 161-166.

5. Rössle M, Gerbes AL. TIPS for the treatment of refractory ascites, hepatorenal syndrome and hepatic hydrothorax: a critical update. Gut. 2010; 59:988-1000. 